PROCEEDINGS OF THE

AMERICAN MATHEMATICAL SOCIETY

Volume 140, Number 6, June 2012, Pages 2183-219

S 0002-9939(2011)11051-7

Article electronically published on October 11, 2011

\title{
A MOMENT ESTIMATE OF THE DERIVATIVE PROCESS IN ROUGH PATH THEORY
}

\author{
YUZURU INAHAMA
}

(Communicated by Richard C. Bradley)

\begin{abstract}
In this paper we prove that the derivative process of a rough differential equation driven by a Brownian rough path has finite $L^{r}$-moment for any $r \geq 1$. This kind of problem is easy in the usual SDE theory, thanks to Burkholder-Davis-Gundy's inequality. In the context of rough path theory, however, it does not seem so obvious.
\end{abstract}

\section{INTRODUCTION}

In stochastic analysis, the derivative process of a given stochastic differential equation (or equivalently, the equation of the stochastic flow) has been studied extensively, because it plays a very important role in various situations. On the other hand, in rough path theory, the derivative process was not studied very much. One reason might be that it has unbounded coefficients. For such a rough differential equation (RDE), proving existence of a solution is in general difficult. The aim of this paper is to prove $L^{r}$-integrability for the first level path of the derivative process for any $r \geq 1$.

Now we will give a more detailed explanation. We consider the following RDE in a Banach setting. Here, $W$ is Brownian rough path and $a$ is an initial value. Then

$$
d Y_{t}=\sigma\left(Y_{t}\right) d W_{t}, \quad Y_{0}=a .
$$

Its derivative equation is given by

$$
d J_{t}=\nabla \sigma\left(Y_{t}\right)\left\langle J_{t} \bullet, d W_{t}\right\rangle, \quad J_{0}=\mathrm{Id} .
$$

Roughly speaking, $J_{t}$ is the derivative of a map $a \mapsto Y_{t}=Y(a)_{t}$. It is known to experts of this research field that a unique solution $(Y, J)$ in the rough path sense exists, although there seems to be no published paper which proves it.

Our main result is as follows (see Theorem 3.7 below for details). Let $2<p<3$ and let $W$ be a Banach space-valued Brownian rough path. Then, under a suitable condition on the coefficient $\sigma$, the $1 / p$-Hölder norm of the first level path $J^{1}$ is $L^{r}$-integrable for any $r \geq 1$.

This kind moment estimate appears in various occasions. In the author's case, when H. Kawabi and he try to prove a stationary phase for solutions of RDEs in a forthcoming paper [9] (which can be regarded as a rough path version of [2]), this type of integrability of the derivative process $J$ is needed.

Received by the editors October 1, 2010 and, in revised form, February 1, 2011.

2010 Mathematics Subject Classification. Primary 60H10; Secondary 60G99.

(C) 2011 American Mathematical Society Reverts to public domain 28 years from publication 
To the author's knowledge, the only expositions which explicitly discuss the derivative equation are Aida's unpublished manuscript [1] and Chapter 11 of Friz and Victoir's book [6. We will basically follow [1], in which Aida established T. Lyons' continuity theorem for $(Y, J)$ and proved the following estimate: If $W$ is controlled by a control function $\omega$, i.e., $\left|W_{s, t}^{i}\right| \leq \omega(s, t)^{i / p}$ for all $0 \leq s \leq t \leq 1$ and $i=1,2$, then $\sup _{t}\left|J_{0, t}^{1}\right| \leq C \exp \left(C \omega(0,1)^{\gamma}\right)$ for some constants $C>0$ and $\gamma \geq 1$. This kind of deterministic argument is of great importance. However, it is not suitable for our purpose, because even if we take $\omega(s, t)=\left\|W^{1}\right\|_{p-v a r ;[s, t]}^{p}+$ $\left\|W^{2}\right\|_{p / 2-v a r ;[s, t]}^{p / 2}$, the right hand side is not integrable. Here, $\|\cdot\|_{p-v a r ;[s, t]}$ is a $p$-variation norm on the subinterval $[s, t]$. Therefore, we need to take a closer look at the arguments in [1].

Remark 1.1. (i) The same results hold for an RDE with a drift term. In such a case, we only need to consider the lift of a "space-time" process $t \mapsto\left(w_{t}, t\right)$ instead of a Brownian rough path. Here, $w$ is Brownian motion (i.e., the first level path of $W)$.

(ii) The author does not know whether the main result is true or not when the driving rough path is a lift of fractional Brownian motion. See Lemma 3.4 and Remark 3.5 below for details.

Remark 1.2. After this work was done, the author was informed that Cass and Lyons 3 also obtained the main result of this paper independently by using a different method (i.e., a Gronwall-type inequality in the rough path context).

\section{SetTing}

Let $(\mathcal{V}, \mathcal{H}, \mu)$ be an abstract Wiener space and let $\left(w_{t}\right)_{0 \leq t \leq 1}$ be Brownian motion on $\mathcal{V}$ associated to $\mu$, which starts at 0 . Let $2<p<3$ and let $G \Omega_{p}(\mathcal{V})$ be the geometric rough path space over $\mathcal{V}$ with $p$-variation norm. (When given the $1 / p$ Hölder norm, the geometric rough path space is denoted by $G \Omega_{1 / p-H l d}(\mathcal{V})$.)

In this article, the time interval is always $[0,1]$ and tensor spaces of Banach spaces are equipped with the projective tensor norm, unless stated otherwise. We basically use the original formulation of rough path theory as in Lyons and Qian [12], although there are a few variants of the theory now.

As in much of the literature on this theory, we use the following notation: 1-variational paths taking their values in a vector space are denoted by small letters such as $x, y, w, j$, etc. The smooth rough paths lying above them (i.e., the natural lifts of them) are denoted by the corresponding capital letters $X, Y, W, J$, etc. Therefore, $X$ may be a general element of the geometric rough path space or the natural lift of the usual 1-variational path $x$, depending on the context.

We denote by $w(m)$ be the $m$ th dyadic approximation of $w$, i.e., the piecewise linear approximation associated to the partition $\left\{0<1 / 2^{m}<2 / 2^{m}<\cdots<\right.$ $\left.\left(2^{m}-1\right) / 2^{m}<1\right\}$. Its lift, i.e., the smooth rough path above $w(m)$, is denoted by $W(m)$ as usual. Unlike the finite dimensional case, the existence of a Brownian rough path is not known. So we set the following assumption:

$$
\lim _{n, m \rightarrow \infty} \mathbb{E}\left[\sup _{0 \leq s \leq t \leq 1}\left|W(m)_{s, t}^{i}-W(n)_{s, t}^{i}\right| \mathcal{V}\right]=0 \text { for } i=1,2 .
$$

The limit is denoted by $W$ and is called a Brownian rough path. (This formulation is used in Dereich 4. The well-known sufficient condition "Exactness" as 
in Definition 4.6.1 of [12 implies (A1). So, we will work under this assumption.) Interestingly, (A1) implies almost sure convergence of $W(m)$, too (see [4).

Let $\mathcal{W}$ be another real Banach space and $\sigma: \mathcal{W} \rightarrow L(\mathcal{V}, \mathcal{W})$ be $C_{b}^{4}$ in the Fréchet sense (i.e., $\nabla^{j} \sigma$ is bounded for $\left.j=0,1, \ldots, 4\right)$. Here, $L(\mathcal{V}, \mathcal{W})$ denotes the set of bounded linear maps from $\mathcal{V}$ to $\mathcal{W}$, which is equipped with the operator norm. Consider the following $\mathrm{RDE}$ for $X \in G \Omega_{p}(\mathcal{V})$ :

$$
d Y_{t}=\sigma\left(Y_{t}\right) d X_{t} \quad \text { with } Y_{0}=a \in \mathcal{W} .
$$

A solution in the rough path sense is $Z=(X, Y) \in G \Omega_{p}(\mathcal{V} \oplus \mathcal{W})$. Its second component $Y \in G \Omega_{p}(\mathcal{W})$ is also called a solution. Under this regularity condition for $\sigma$, this RDE has a unique solution. So, $X \mapsto Y(=: \Phi(X))$ defines a map, which is called an Itô map. By T. Lyons' continuity theorem, $\Phi: G \Omega_{p}(\mathcal{V}) \rightarrow G \Omega_{p}(\mathcal{W})$ is (locally Lipschitz) continuous.

Aida 11 gave a rather quantitative estimate for the growth of the solution and the local Lipschitz constant for two solutions. If $X$ is controlled by a control function $\omega$ (that is, $\left|X_{s, t}^{i}\right| \leq \omega(s, t)^{i / p}$ for all $s \leq t$ and $\left.i=1,2\right)$, then $Z=(X, Y)$ is controlled by a control function $\hat{\omega}$ of the form $\hat{\omega}(s, t)=C\left(1+\omega(0,1)^{\gamma}\right) \omega(s, t)$ with certain positive constants $C, \gamma$, which are independent of the initial value $a$.

Adding to RDE (2.3), we also consider the following "derivative equation":

$$
d J_{t}=\nabla \sigma\left(Y_{t}\right)\left\langle J_{t} \bullet, d X_{t}\right\rangle \quad \text { with } J_{0}=\operatorname{Id}_{\mathcal{W}} .
$$

Notice that a solution $J$ takes its values in $L(\mathcal{W}):=L(\mathcal{W}, \mathcal{W})$ and that $\nabla \sigma\left(Y_{t}\right)$ is a bounded bilinear map from $\mathcal{W} \times \mathcal{V}$ to $\mathcal{W}$.

The RDEs (2.1) and (2.2) are obviously equivalent to considering the following RDE:

$$
\begin{aligned}
d Y_{t} & =\sigma\left(a+Y_{t}\right) d X_{t} \quad \text { with } Y_{0}=0 \in \mathcal{W}, \\
d J_{t} & =\nabla \sigma\left(a+Y_{t}\right)\left\langle\left(\operatorname{Id}_{\mathcal{W}}+J_{t}\right) \bullet, d X_{t}\right\rangle \quad \text { with } J_{0}=0 .
\end{aligned}
$$

We will basically study the RDEs (2.3) and (2.4). (More precisely, the shifted equations as above are the definition of an RDE with a nonzero initial condition.)

$\mathrm{RDE}(2.4)$ can be written in a simpler way as follows:

$$
d J_{t}=d\left(\operatorname{Id}_{\mathcal{W}}+J_{t}\right)=\left(d M_{t}\right) \cdot\left(\operatorname{Id}_{\mathcal{W}}+J_{t}\right) \quad \text { with } J_{0}=0,
$$

where $M$ is an $L(\mathcal{W})$-valued rough path, which is given by

$$
M_{t}:=\int_{0}^{t} \nabla \sigma\left(a+Y_{u}\right)\left\langle\cdot, d X_{u}\right\rangle .
$$

Note that the map $\Lambda: \mathcal{V} \oplus \mathcal{W} \rightarrow L(\mathcal{V} \oplus \mathcal{W}, L(\mathcal{W}))$ defined by

$$
\Lambda(\xi, \eta)\left\langle\xi^{\prime}, \eta^{\prime}\right\rangle=\nabla \sigma(\eta)\left\langle\cdot, \xi^{\prime}\right\rangle \in L(\mathcal{W}) \text { with }(\xi, \eta),\left(\xi^{\prime}, \eta^{\prime}\right) \in \mathcal{V} \oplus \mathcal{W}
$$

is $C_{b}^{3}$ and the right hand side of (2.5) is well-defined as a rough path integral. So, if $X$ is controlled by $\omega$, then the right hand side of (2.6) is a rough integral and $M$ is controlled by $\omega^{\prime}(s, t)=C^{\prime}\left(1+\omega(0,1)^{\gamma^{\prime}}\right) \omega(s, t)$, with certain positive constants $C^{\prime}, \gamma^{\prime}$, which are independent of the initial value $a$.

The RDEs (2.3) and (2.4) combined as one have a linear growth coefficient. So, it is not at all clear whether the solution $J$ exists or not. (It is not very difficult to show the uniqueness if a (global) solution exists.) There are some papers (e.g., Lejay [11) which study Lyons' continuity theorem for RDEs with unbounded coefficients. But, the case of the derivative equations (2.3)-(2.4) does not seem to be included. 
In solving an RDE with an unbounded coefficient, the most difficult part is always how to control the first level path of a solution. In this case, however, thanks to the special form in (2.5) and the series representation (2.7) below, it is possible to prove the existence of a unique solution $(Y, J)$.

Let us recall how this is solved in [1]:

(Step 1) Let us first consider the case when $X$ is a smooth rough path lying above $x \in C_{0}^{1-v a r}([0,1], \mathcal{V})$. Then, the ODEs (2.3) and (2.4) have a unique solution $t \mapsto\left(y_{t}, j_{t}\right)$ in the 1 -variational sense. Moreover, it is well known that $j_{t}$ can be written explicitly as follows:

$$
\mathrm{Id}+j_{t}=\left(\mathrm{Id}+j_{s}\right)\left(\mathrm{Id}+\sum_{k=1}^{\infty} A_{k ; s, t}\right) \quad(s \leq t),
$$

where $A_{k}$ is given by

$$
A_{k ; s, t}=\int_{s<t_{1}<\cdots<t_{k}<t} d M_{t_{k}} \cdots d M_{t_{1}},
$$

and $M_{t}$ on the right hand side is given by (2.6) in the 1-variational sense (with $X$ and $Y$ being replaced with $x$ and $y$, resp.). Notice the order of the product of the $M_{t_{j}}$ 's on the right hand side.

(Step 2) Fortunately, $A_{k}$ is written in the form of an interated integral. So, the series representation in (2.7)-(2.8) fits well with rough path theory. The following argument is quite similar to the "fundamental theorem of rough path theory" (Theorem 3.1.2, [12]), which states that one can obtain the $i$ th level path $(i \geq 3)$ from the first and the second level paths.

Note also that, in the same way as in the fundamental theorem, the map $x \mapsto M \mapsto j$ extends continuously with respect to the topology of $G \Omega_{p}(\mathcal{V})$.

(Step 3) Suppose that $x \in C_{0}^{1-v a r}([0,1], \mathcal{V})$ satisfies that $\left\|X^{1}\right\|_{p-v a r}^{p}+\left\|X^{2}\right\|_{p / 2-v a r}^{p / 2}$ $\leq R$ for $R>0$. Then, it is shown that $\sup _{0 \leq t \leq 1}\left|j_{t}\right| \leq C_{R}<\infty$ for some positive constant $C_{R}$.

So, when we try to solve the RDEs (2.3)-(2.4) for such an $x$, the (local) solution $(Y, J)$ coincides with (the lift of) the solution $(y, j)$ in the usual sense and it (= its first level path) does not get out of a large ball of radius $C_{R}^{\prime}>0$. It is also shown in [1] that, for such an $x$ and its lift $X$, the Lipschitz property for the map holds:

$$
X \mapsto(Y, J) \in G \Omega_{p}(\mathcal{W} \oplus L(\mathcal{W})) .
$$

Therefore, this map naturally extends to one from $\left\{X \in G \Omega_{p}(\mathcal{V}) \mid\left\|X^{1}\right\|_{p-v a r}^{p}+\right.$ $\left.\left\|X^{2}\right\|_{p / 2-v a r}^{p / 2} \leq R\right\}$ to $G \Omega_{p}(\mathcal{W} \oplus L(\mathcal{W}))$. Since $R>0$ is arbitrary, this map is defined for any $X \in G \Omega_{p}(\mathcal{V})$. Summing up, we have the following proposition in [1]. (Precisely speaking, a more qualitative result is shown in [1.)

Proposition 2.1. The RDEs (2.3) -(2.2) have a unique solution for any $X$, and the map

$$
X \in G \Omega_{p}(\mathcal{V}) \mapsto(Y, J) \in G \Omega_{p}(\mathcal{W} \oplus L(\mathcal{W}))
$$

is (locally Lipschitz) continuous. Moreover, $J^{1}$, i.e., the first level path of $J$, admits a series representation as in (2.7)-(2.8). 


\section{Moment estimate of $J^{1}$}

In this section we prove that the $L^{r}$-moment of $J^{1}$ is finite when $X=W$ by using the series representation in (2.7) (2.8).

Let $M=\left(M^{1}, M^{2}\right) \in G \Omega_{p}(L(\mathcal{W}))$. Then, by the fundamental theorem of rough path theory, we can construct $M^{3}, M^{4}, \ldots$. When $M$ is a smooth rough path lying above $m$, then $M^{k}$ coincides with the iterated Stieltjes integral, i.e.,

$$
M_{s, t}^{k}=\int_{s<t_{1}<\cdots<t_{k}<t} d m_{t_{1}} \otimes \cdots \otimes d m_{t_{k}} \quad(k \geq 1) .
$$

$A_{k}$ in (2.8) is dominated by $M^{k}$.

Lemma 3.1. Set

$$
A_{k: s, t}=\int_{s<t_{1}<\cdots<t_{k}<t} d m_{t_{k}} \cdots d m_{t_{1}}
$$

for a smooth rough path $M=\left(M^{1}, M^{2}\right) \in G \Omega_{p}(L(\mathcal{W}))$ lying above $m$. Then, $M \mapsto$ $A_{k: s, t}$ extends to a continuous map from $G \Omega_{p}(L(\mathcal{W}))$ and the following inequality holds:

$$
\left|A_{k: s, t}\right| \leq\left|M_{s, t}^{k}\right| \quad \text { for all } k \text { and } s \leq t
$$

Proof. Set $T: L(\mathcal{W})^{\otimes k} \rightarrow L(\mathcal{W})$ by $T\left(a_{1} \otimes \cdots \otimes a_{k}\right)=a_{k} \cdots a_{1}$. By a basic property of the projective tensor norm, the operator norm of $T$ is 1 . Noting that $A_{k: s, t}=T\left(M_{s, t}^{k}\right)$, we can easily prove the lemma.

It is well known how fast the $L^{r}$-norm of $W^{i}(i=1,2)$ grows as $r \rightarrow \infty$, from which one can obtain the growth of $M^{i}(i=1,2)$.

Lemma 3.2. There is a positive constant $C$ such that

$$
\mathbb{E}\left[\left\|W^{i}\right\|_{i / p-H l d}^{r}\right]^{1 / r} \leq C r^{i / 2} \quad \text { for all } r \geq 1 \text { and } i=1,2 .
$$

Proof. Recall that a Fernique-type theorem holds for a Brownian rough path: there exists a positive constant $\beta$ such that $\mathbb{E}\left[\exp \left(\beta\left(\left\|W^{1}\right\|_{1 / p-H l d}+\left\|W^{2}\right\|_{2 / p-H l d}^{1 / 2}\right)^{2}\right)\right]<$ $\infty$. (This type of theorem was first shown in [10]. For similar results, see also [8, 13, 5] for instance.) Dereich 44 gives a proof under Assumption (A1).

We will show that, in general, if a random variable $Z \geq 0$ is defined on a certain probability space with Fernique-type integrability condition $\mathbb{E}\left[e^{\beta Z^{2}}\right]<\infty$, then $\|Z\|_{L^{r}}=O(\sqrt{r})$. (In the following, we assume $\beta>1 / 2$ for simplicity. Otherwise, we take a constant multiple of $Z$ instead of $Z$ itself.) By Chebychev's inequality, there exists a constant $C>0$ such that $\mathbb{P}(Z \geq \eta) \leq C e^{-\beta \eta^{2}}$ for all $\eta>0$. Let $\varepsilon>0$ be sufficiently small so that $\alpha:=\beta-\varepsilon>1 / 2$.

Then, for $r \geq 1$,

$$
\begin{aligned}
\mathbb{E}\left[Z^{r}\right] & \leq \sum_{n=0}^{\infty}(n+1)^{r} \mathbb{P}(n<Z \leq n+1) \\
& \leq C \sum_{n=0}^{\infty}(n+1)^{r} e^{-\beta n^{2}} \\
& \leq C r^{r / 2} \sum_{n=0}^{\infty} \exp \left(-\frac{r \log r}{2}+r \log (n+1)-\alpha n^{2}\right) e^{-\varepsilon n^{2}} .
\end{aligned}
$$


So, it suffices to show that $f(n, r):=-\frac{r \log r}{2}+r \log (n+1)-\alpha n^{2}$ is bounded from above. $\partial f / \partial n(n, r)=-2 \alpha n+r /(n+1)$. It is easy to see that, as a function of $n$, $f$ takes its maximum at $n=\left(-1+\sqrt{1+2 \alpha^{-1} r}\right) / 2$. Hence,

$$
f(n, r) \leq-\frac{r \log r}{2}+r \log \left(\frac{1+\sqrt{1+2 \alpha^{-1} r}}{2}\right)-\alpha\left(\frac{-1+\sqrt{1+2 \alpha^{-1} r}}{2}\right)^{2} .
$$

It is easy to see that, as $r \rightarrow \infty$,

$$
\alpha\left(\frac{-1+\sqrt{1+2 \alpha^{-1} r}}{2}\right)^{2} \approx \frac{r}{2}, \quad \frac{1+\sqrt{1+2 \alpha^{-1} r}}{2} \approx \sqrt{\frac{r}{2 \alpha}} .
$$

Take $\delta>0$ so small that $\log (1+\delta)<1 / 4$. There exists $r_{0}>0$ such that, for all $r \geq r_{0}$

$$
\alpha\left(\frac{-1+\sqrt{1+2 \alpha^{-1} r}}{2}\right)^{2} \geq \frac{r}{4}, \quad \log \left(\frac{1+\sqrt{1+2 \alpha^{-1} r}}{2}\right) \leq \log \left((1+\delta) \sqrt{\frac{r}{2 \alpha}}\right) .
$$

So, the right hand side of (3.2) is dominated by

$$
\left.r\left(\log (1+\delta)-\frac{1}{2} \log (2 \alpha)-\frac{1}{4}\right) \leq 0 \quad \text { (for any } r \geq r_{0}\right)
$$

which implies that the right hand side of (3.2) is bounded from above.

Lemma 3.3. When $X=W$ (i.e., Brownian rough path), there are positive constants $c$ and $\alpha$ independent of $r \geq 1$, which satisfy that, for all $s \leq t$,

$$
\begin{aligned}
& \mathbb{E}\left[\left|M_{s, t}^{1}\right|^{r}\right]^{1 / r} \leq \frac{r^{\alpha}|c(t-s)|^{1 / p}}{\beta_{p}(1 / p) !}, \\
& \mathbb{E}\left[\left|M_{s, t}^{2}\right|^{r}\right]^{1 / r} \leq \frac{r^{2 \alpha}|c(t-s)|^{2 / p}}{\beta_{p}(2 / p) !} .
\end{aligned}
$$

Here, $\beta_{p}$ is a positive constant such that

$$
\beta_{p} \geq p^{2}\left(1+\sum_{j=3}^{\infty}\left(\frac{2}{j-2}\right)^{3 / p}\right) .
$$

(Any such positive constant which satisfies the above inequality will do.)

Proof. We can choose $\omega(s, t)=\left(\left\|W^{1}\right\|_{1 / p-H l d}^{p}+\left\|W^{2}\right\|_{2 / p-H l d}^{p / 2}\right)(t-s)$ as a control of $W$. Then, as is explained in (2.3), $M$ is controlled by $\omega^{\prime}(s, t)=C^{\prime}(1+$ $\left.\omega(0,1)^{\gamma^{\prime}}\right) \omega(s, t)$, where $C^{\prime}, \gamma^{\prime}$ are positive constants. Now, by using inequality (3.1) in Lemma 3.2 and choosing a suitable constant $c>0$, we can see that the lemma holds for $2 \alpha=1+\left(\gamma^{\prime} / p\right)$.

Proposition 3.4. Let $X=W$ and set $\eta(s, t)=c(t-s)$, where $c$ is a positive constant as in Lemma 3.3 above. Then, for all $k=1,2,3, \ldots, r \geq 1$, and $s \leq t$, it follows that

$$
\mathbb{E}\left[\left|M_{s, t}^{k}\right|^{r}\right]^{1 / r} \leq \frac{r^{k \alpha} \eta(s, t)^{k / p}}{\beta_{p}(k / p) !}
$$

Proof. We use induction. The cases $k=1,2$ were already shown. Assume the inequality (3.3) up to $k-1$ and let us prove (3.3) for $k$.

Let us write $M_{s, t}^{j}=M(a, X)_{s, t}^{j}$, where $X$ is the driving rough path and $a \in \mathcal{W}$ is the initial condition of the RDE. For a dyadic rational number $u \in[0,1]$, we set $\tilde{w}_{t}=w_{u+t}-w_{u}$ for $0 \leq t \leq 1-u$. Since $\tilde{w}$ and $\left\{w_{s}\right\}_{0 \leq s \leq u}$ are independent, 
$\tilde{W}$, the lift of $\tilde{w}$, and $\left\{W_{s, s^{\prime}}\right\}_{0 \leq s \leq s^{\prime} \leq u}$ are independent. For $u<t, M(a, W)_{u, t}^{j}=$ $M\left(a+Y(a, W)_{0, u}^{1}, \tilde{W}\right)_{0, t-u}^{j}$.

From this independence, we see that, for $1 \leq j \leq k-1$,

$$
\begin{aligned}
\mathbb{E}\left[\left|M_{s, u}^{j} \otimes M_{u, t}^{k-j}\right|^{r}\right]^{1 / r} & \leq \mathbb{E}\left[\left|M(a, W)_{s, u}^{j}\right|^{r} \cdot \tilde{\mathbb{E}}\left[\left|M\left(a+Y(a, W)_{0, u}^{1}, \tilde{W}\right)_{0, t-u}^{k-j}\right|^{r}\right]\right]^{1 / r} \\
& \leq \mathbb{E}\left[\left|M(a, W)_{s, u}^{j}\right|^{r} \cdot\left(\frac{r^{(k-j) \alpha} \eta(0, t-u)^{(k-j) / p}}{\beta_{p}((k-j) / p) !}\right)^{r}\right]^{1 / r} \\
& \leq \frac{r^{j \alpha} \eta(s, u)^{j / p}}{\beta_{p}(j / p) !} \cdot \frac{r^{(k-j) \alpha} \eta(u, t)^{(k-j) / p}}{\beta_{p}((k-j) / p) !} .
\end{aligned}
$$

Here, $\tilde{\mathbb{E}}$ denotes the expectation with respect to $\tilde{W}$. By taking the limit, we can easily see that (3.4) holds for any $s<u<t$.

Let $\mathcal{P}=\left\{s=t_{0}<t_{1}<\cdots<t_{L}=t\right\}$ be a partition of the interval $[s, t]$ and set

$$
M_{s, t}^{k}(\mathcal{P}):=\sum_{j=1}^{k-1} \sum_{i=1}^{L} M_{s, t_{i-1}}^{j} \otimes M_{t_{i-1}, t_{i}}^{k-j} .
$$

Recall that, by the fundamental theorem of rough path theory, $M_{s, t}^{k}$ is obtained as the limit $M_{s, t}^{k}=\lim _{|\mathcal{P}| \searrow 0} M_{s, t}^{k}(\mathcal{P})$.

It is well known that there exists $t_{l} \in \mathcal{P}$ such that $\eta\left(t_{l-1}, t_{l+1}\right) \leq 2 \eta(s, t) /(L-1)$ if $L \geq 3$. (When $L=2$, we have a trivial (in)equality.) By straightforward computation,

$$
M_{s, t}^{k}(\mathcal{P})-M_{s, t}^{k}\left(\mathcal{P} \backslash\left\{t_{l}\right\}\right)=\sum_{j=1}^{k-1} M_{t_{l-1}, t_{l}}^{j} \otimes M_{t_{l}, t_{l+1}}^{k-j} .
$$

Now, we use the binomial inequality (also known as the neo-classical inequality), which states that, for any $p \geq 1, a, b \in[0, \infty), k=1,2, \ldots$,

$$
\sum_{j=0}^{k} \frac{a^{j / p} b^{(k-j) / p}}{\left(\frac{j}{p}\right) !\left(\frac{k-j}{p}\right) !} \leq p^{2} \frac{(a+b)^{k / p}}{\left(\frac{k}{p}\right) !} .
$$

(By the way, Hara and Hino [7] recently proved that the best constant on the right hand side of (3.6) is $p$, not $p^{2}$.)

By taking the $L^{r}$-norm of (3.5) when $X=W$, we see that

$$
\begin{aligned}
\left\|M_{s, t}^{k}(\mathcal{P})-M_{s, t}^{k}\left(\mathcal{P} \backslash\left\{t_{l}\right\}\right)\right\|_{L^{r}} & \leq \sum_{j=1}^{k-1}\left\|M_{t_{l-1}, t_{l}}^{j} \otimes M_{t_{l}, t_{l+1}}^{k-j}\right\|_{L^{r}} \\
& \leq \sum_{j=1}^{k-1} \frac{r^{j \alpha} \eta\left(t_{l-1}, t_{l}\right)^{j / p}}{\beta_{p}(j / p) !} \cdot \frac{r^{(k-j) \alpha} \eta\left(t_{l}, t_{l+1}\right)^{(k-j) / p}}{\beta_{p}((k-j) / p) !} \\
& \leq\left(\frac{p}{\beta_{p}}\right)^{2} \frac{r^{k \alpha} \eta\left(t_{l-1}, t_{l+1}\right)^{k / p}}{(k / p) !} \\
& \leq\left(\frac{p}{\beta_{p}}\right)^{2}\left(\frac{2}{L-1}\right)^{k / p} \frac{r^{k \alpha} \eta(s, t)^{k / p}}{(k / p) !}
\end{aligned}
$$


for $L \geq 3$. Hence, we see from the definition of $\beta_{p}$ that

$$
\begin{aligned}
\left\|M_{s, t}^{k}(\mathcal{P})\right\|_{L^{r}} & \leq\left(\frac{p}{\beta_{p}}\right)^{2}\left(1+\sum_{L=3}^{\infty}\left(\frac{2}{L-1}\right)^{k / p}\right) \frac{r^{k \alpha} \eta(s, t)^{k / p}}{(k / p) !} \\
& \leq\left(\frac{p}{\beta_{p}}\right)^{2}\left(1+\sum_{L=3}^{\infty}\left(\frac{2}{L-1}\right)^{3 / p}\right) \frac{r^{k \alpha} \eta(s, t)^{k / p}}{(k / p) !} \leq \frac{r^{k \alpha} \eta(s, t)^{k / p}}{\beta_{p}(k / p) !}
\end{aligned}
$$

which implies that

$$
\left\|M_{s, t}^{k}\right\|_{L^{r}} \leq \frac{r^{k \alpha} \eta(s, t)^{k / p}}{\beta_{p}(k / p) !}=\frac{\left\{r^{\alpha} C^{1 / p}\right\}^{k}(t-s)^{k / p}}{\beta_{p}(k / p) !} .
$$

This completes the proof.

Remark 3.5. Notice that, in the proof of the above lemma, we used the independent increment property of Brownian motion. So, if the driving process is replaced with fractional Brownian motion, this proof fails.

Proposition 3.6. There exists a positive constant $C=C_{r}$ which is independent of $s, t$ such that $\sum_{k=1}^{\infty}\left\|A_{k: s, t}\right\|_{L^{r}} \leq C(t-s)^{1 / p}$ for all $s<t$ and $\left\|\operatorname{Id}_{\mathcal{W}}+J_{0, t}^{1}\right\|_{L^{r}} \leq C$ for all $t$.

Proof. Recall Stirling's formula:

$$
\lambda !:=\Gamma(\lambda+1) \sim \sqrt{2 \pi \lambda} \lambda^{\lambda} e^{-\lambda} \quad \text { as } \lambda \nearrow \infty .
$$

Then, the first estimate holds for $C=\sum_{k}\left\{r^{\alpha} C^{1 / p}\right\}^{k} /\left\{\beta_{p}(k / p) !\right\}$. The second estimate is clear from the first one since $\operatorname{Id}_{\mathcal{W}}+J_{0, t}^{1}=\operatorname{Id}_{\mathcal{W}}+\sum_{k=1}^{\infty} A_{k: 0, t}$.

Set

$$
\|\psi\|_{m, \theta}:=\left(\iint_{0<s<t<1} \frac{\left\|\psi_{t}-\psi_{s}\right\|_{\mathcal{W}}^{m}}{|t-s|^{2+m \theta}} d s d t\right)^{1 / m}, \quad m \in[1, \infty), \theta \in(0,1],
$$

for a continuous path $\psi$ in the usual sesne, which takes its values in a Banach space $\mathcal{W}$ and starts at 0 . It is well known that this norm is stronger than the Hölder norm; there exists a positive constant $C_{m, \theta}$ such that, for all $\psi,\|\psi\|_{1 / p-H l d} \leq$ $C_{m, \theta}\|\psi\|_{m, 1 / p}$.

Now we give the main result of this paper, which states that the first level path of the derivative equation (2.2) is $L^{r}$-integrable for any $r \geq 1$, provided $X=W$.

Theorem 3.7. Assume (A1) and $\sigma: \mathcal{W} \rightarrow L(\mathcal{V}, \mathcal{W})$ be $C_{b}^{4}$. Consider the RDEs (2.3) and (2.4) with the driving rough path being the Brownian rough path, i.e., $X=W$. We denote by $J^{1}$ the first level path of the solution of the second RDE (2.4). Then, $\mathbb{E}\left[\left\|J^{1}\right\|_{1 / p-H l d}^{r}\right]<\infty$ for any $r \geq 1$.

Proof. From the series representation (2.7), it is clear that

$$
J_{s, t}^{1}=\left(\mathrm{Id}+J_{0, t}^{1}\right) \sum_{k=1}^{\infty} A_{k: s, t} .
$$

We obtain from Proposition 3.6 that

$$
\left\|J_{s, t}^{1}\right\|_{L^{r}}=\left\|\mathrm{Id}+J_{0, t}^{1}\right\|_{L^{2 r}} \cdot\left\|\sum_{k=1}^{\infty} A_{k: s, t}\right\|_{L^{2 r}} \leq C \sqrt{t-s} .
$$


If $r>1$ is so large that $2+r\left(p^{-1}-2^{-1}\right)<1$, then we can easily see that

$$
\begin{aligned}
\mathbb{E}\left[\left\|J^{1}\right\|_{1 / p-H l d}^{r}\right] & \leq \mathbb{E}\left[\left\|J^{1}\right\|_{r, 1 / p}^{r}\right] \leq \iint_{0<s<t<1} \frac{\mathbb{E}\left[\left|J_{s, t}^{1}\right|^{r}\right]}{|t-s|^{2+r / p}} d s d t \\
& \leq C \iint_{0<s<t<1} \frac{1}{|t-s|^{2+r(1 / p-1 / 2)}} d s d t<\infty .
\end{aligned}
$$

Thus, we have shown the theorem.

Remark 3.8. The inverse of the flow $\left(\operatorname{Id}+J_{t}\right)^{-1}$ is also $L^{r}$-integrable for any $r \geq 1$. For example, instead of (2.5) and (2.7), we can easily obtain

$$
d\left(\operatorname{Id}+J_{t}\right)^{-1}=-\left(\operatorname{Id}_{\mathcal{W}}+J_{t}\right)^{-1} \cdot\left(d M_{t}\right)
$$

and, for $s \leq t$,

$$
\left(\mathrm{Id}+j_{t}\right)^{-1}=\left(\mathrm{Id}+\sum_{k=1}^{\infty}(-1)^{k} \int_{s<t_{1}<\cdots<t_{k}<t} d m_{t_{1}} \cdots d m_{t_{k}}\right)\left(\mathrm{Id}+j_{s}\right)^{-1}
$$

respectively. Therefore, we may use essentially the same argument.

\section{REFERENCES}

[1] Aida, S.; T. Lyons no renzokusei teiri no shoumei ni tsuite (in Japanese: "On the proof of T. Lyons' continuity theorem"), preprint (2006).

[2] Ben Arous, G.; Methods de Laplace et de la phase stationnaire sur l'espace de Wiener. Stochastics 25 (1988), no. 3, 125-153. MR999365 (91h:60070)

[3] Cass, T.; Lyons, T.; Evolving communities with individual preference, preprint (2010).

[4] Dereich, S.; Rough paths analysis of general Banach space-valued Wiener processes. J. Funct. Anal. 258 (2010), no. 9, 2910-2936. MR2595729

[5] Friz, P.; Oberhauser, H.; A generalized Fernique theorem and applications, Proc. Amer. Math. Soc. 138 (2010), 3679-3688. MR2661566

[6] Friz, P.; Victoir, N.; Multidimensional stochastic processes as rough paths, Cambridge University Press, 2010. MR2604669

[7] Hara, K.; Hino, M.; Fractional order Taylor's series and the neo-classical inequality, Bull. Lond. Math. Soc. 42 (2010), 467-477. MR.2651942

[8] Inahama, Y.; Kawabi, H.; Large deviations for heat kernel measures on loop spaces via rough paths, J. London Math. Soc. (2) 73 (2006), no. 3, 797-816. MR2241981(2007i:58047)

[9] Inahama, Y.; Kawabi, H.; Stationary phase for solutions of rough differential equations. In preparation.

[10] Ledoux, M.; Qian, Z.; Zhang, T.; Large deviations and support theorem for diffusion processes via rough paths, Stochastic Process. Appl. 102 (2002), no. 2, 265-283. MR.1935127 (2003m:60152)

[11] Lejay, A.; On rough differential equations. Electron. J. Probab. 14 (2009), no. 12, 341-364. MR2480544 (2010i:60177)

[12] Lyons, T.; Qian, Z.; System control and rough paths. Oxford Math. Monographs, Oxford Science Publications, Oxford University Press, Oxford, 2002. MR2036784 (2005f:93001)

[13] Millet, A.; Sanz-Solé, M.; Large deviations for rough paths of the fractional Brownian motion, Ann. Inst. H. Poincaré Probab. Statist. 42 (2006), no. 2, 245-271. MR2199801(2007e:60027)

Graduate School of Mathematics, Nagoya University, Furocho, Chikusa-ku, Nagoya 464-8602, JAPAN

E-mail address: inahama@math.nagoya-u.ac.jp 